\title{
Formalización de un modelo de trabajo con empresas en una carrera de ingeniería
}

\author{
Formalizing a working model with businesses in an engineering career
}

\author{
Rodolfo Schmal Simón ${ }^{1} \quad$ Sabino Rivero Flores ${ }^{1} \quad$ Cristian Vidal Silva ${ }^{2}$
}

Recibido 4 de septiembre de 2014, aceptado 26 de marzo de 2015

Received: September4, 2014 Accepted: March 26, 2015

\begin{abstract}
RESUMEN
La carrera de Ingeniería en Informática Empresarial de la Universidad de Talca, inicia sus actividades en el año 2005, siendo acreditada por 5 años el año 2012. Entre sus fortalezas destaca su vinculación con el sector productivo a partir del desarrollo de proyectos en empresas por parte de los alumnos. Sin embargo, la informalidad con que se ha implementado esta interacción, ha dificultado la gestión global de los proyectos por la diversidad de alcances, grados de complejidad de los proyectos y calidad de sus productos, lo que puede afectar la relación cultivada con las empresas y que es el sello distintivo de la carrera. Actualmente, la carrera tiene 225 alumnos, quienes ejecutan más de 100 proyectos anuales realizados en más de 60 empresas, tanto pequeñas como grandes.
\end{abstract}

Para resolver el problema expuesto, se ha planteado formalizar un modelo de trabajo que permita establecer y controlar la apertura, cierre, y unificar criterios en torno al alcance y complejidad de los proyectos, y de la calidad de los productos y plazos esperados en beneficio de los distintos actores (empresas, alumnos y profesores).

Como resultado se tiene un modelo de trabajo con empresas basado en las actividades que producen los cambios de estado en los proyectos y que se expresa en diagramas de máquina de estados, de proveedoresentradas-procesos-salidas-consumidores (SIPOC), y de procesos.

Con la implementación del modelo se aspira dar sustentabilidad a una forma de trabajar con las empresas que ha sido apreciada por alumnos, egresados y empleadores por facilitar una temprana inserción laboral.

Palabras clave: Proyectos con empresas, modelo de trabajo, vinculación con el medio, formalización de procesos.

\begin{abstract}
The program of Business Informatics Engineering in the Universidad de Talca, started its activities in 2005 and was accredited in 2012 for a period of 5 years. Among its strengths is the link with the production sector established by the development of projects in the businesses by the students. However, the informality with which it has implemented this interaction has hampered the overall management of the projects due to the diversity of the scopes, the degree of complexity of the projects, and the quality of the products that can affect the cultivated relationship with the businesses that is unique trademark of the program. Currently, the program has 225 students who annually conduct more than 100 projects in about 60 companies including both small and large companies.
\end{abstract}

\footnotetext{
1 Escuela de Ingeniería en Informática Empresarial. Universidad de Talca. Avda.Lircay s/n. Talca, Chile. E-mail: rschmal@utalca.cl; srivero@utalca.cl

2 Departamento de Computación e Informática. Facultad de Ingeniería. Universidad de Playa Ancha. Valparaíso, Chile. E-mail: cristian.vidal@upla.cl
} 
To solve this highlighted problem, it has been proposed to formalize a working model to establish and control the opening, closing, and unifying criteria of the scope and complexity of the projects, and of the quality of the products and the expected benefit for the stakeholders (businesses, students, and professors).

As the result, we have a model of working with businesses based on the activities that produce changes in the status of the projects, expressed in the diagrams of the finite-state machine, of the tool SIPOC (suppliers, inputs, process, outputs, and customers), and of the processes.

With the implementation of the model, we aim to achieve sustainability for the opportunity of working with businesses that have been appreciated by students, alumni, and employers to facilitate early employment.

Keywords: Projects with businesses, model of working, link to society, formalization of processes.

\section{INTRODUCCIÓN}

El año 2004, la Universidad de Talca, creó oficialmente la carrera de Ingeniería en Informática Empresarial (IIE) para recibir en el año 2005 a su primera cohorte de alumnos. La carrera fue creada para formar un profesional capaz de agregar valor a las organizaciones por la vía de incorporar las nuevas tecnologías de información y comunicación en la gestión [1].

Simultáneamente, la universidad se propuso implementar un modelo educativo basado en competencias, concepto que encierra la movilización de saberes no solo cognitivos, sino que procedimentales $\mathrm{y}$ actitudinales, asociados al saber hacer, ser y estar [2-4]. Es por ello que se planteó:

1. La necesidad de establecer una metodología de enseñanza basada en: aprendizajes sobre experiencias concretas, esto es, aprender haciendo (learning by doing); $\mathrm{y}$

2. Aplicar la innovación como concepto que atraviese toda la carrera con miras a alentar nuevas iniciativas, nuevas miradas; valorar el aprendizaje en distintos contextos (empresas, carreras, universidades y/o países).

Lo señalado, llevó a implementar un esquema formativo con fuerte participación de las empresas mediante el desarrollo de proyectos reales [5].

Esta filosofía bajo la cual se estructuró la carrera de IIE se apoya en lo que se denomina educación cooperativa o sistema dual, donde el proceso formativo se da en las universidades y en las empresas
[6-8]. Bajo este sistema los estudiantes alternan sus espacios de aprendizaje entre la universidad y la empresa en distintos períodos académicos y donde las responsabilidades y costos son compartidos. Con ello se logra una formación profesional que incluye experiencias de trabajos reales en empresas facilitando una temprana inserción laboral a los egresados. Cabe destacar que en Chile, por lo general, las carreras de ingeniería sólo incluyen un período de práctica profesional en sus planes de estudio como medio de interacción de alumnos con empresas en proyectos reales.

El fundamento de la filosofía adoptada reside en el aprendizaje significativo y las competencias transversales o genéricas que se logran en los estudiantes y que se relacionan con las actitudes que están siendo crecientemente demandadas por las empresas [9-11].

En la carrera de IIE este sistema se aplica con dos variantes: una, que el aprendizaje en la universidad y en la empresa se da en un mismo período académico; y dos, que la responsabilidad y los costos de su implementación es asumida por los estudiantes y la universidad. Estas diferencias se deben a que en Chile no existe el concepto de aprendiz al interior de las empresas, ni una legislación sobre la materia. En América Latina, Montoya y Aguilar destacan que en Colombia sí existe una legislación que regula estas actividades realizadas por estudiantes en las empresas [12].

La elección de este modelo de trabajo se explica porque los estudiantes se sienten más motivados por la vía de aprender haciendo, además de visualizar que una vez egresados, ven facilitada su empleabilidad 
laboral [13-15]. Encuestas aplicadas, anualmente, a estudiantes de $4^{\circ}$ año de la carrera confirman lo expuesto al señalar el trabajo práctico en empresas como el atributo más positivo que posee la carrera.

Su implementación se ha dado a través de grupos de estudiantes que se conforman para desarrollar proyectos reales en empresas, al menos en un módulo por nivel ${ }^{3}$. Estos se complementan con módulos integradores que se desarrollan al término del tercer y cuarto año bajo la modalidad de consultorías, concepto que si bien se asocia a lo que habitualmente se entiende por prácticas, tiene una connotación distinta dada por la responsabilidad que adquieren los estudiantes y los profesores involucrados, y porque los trabajos a realizar se corresponden con la formación recibida a la fecha [16].

Este modelo de trabajo ha sido acogido y valorado positivamente por los estudiantes y egresados de la carrera por facilitar su inserción laboral. Durante el proceso de acreditación al que se sometió la carrera en el año 2012, y donde se obtuvo una acreditación por 5 años, se valoró su existencia, concepción, diseño e implementación. Sin embargo, se observó su informalidad, porque obstaculiza una apropiada gestión de los alcances de proyectos con complejidades disímiles que generan un desbalance en el tiempo de dedicación que tienen los proyectos ya sea en un mismo módulo, como en distintos módulos con igual número de créditos.

Adicionalmente, la informalidad ha impedido el abordaje de proyectos de mayor envergadura que requieren ser descompuestos en proyectos de menor alcance para que puedan ser desarrollados por distintos grupos y/o en distintos módulos.

La informalidad también ha generado que la información disponible respecto de los proyectos se encuentre incompleta, además de dificultar la recolección de evidencias de los resultados alcanzados. Lo expuesto ha afectado la gestión de los proyectos a nivel de la Escuela y el logro

3 Administración en primer año; Análisis y Diseño Lógico de Sistemas, Análisis del Entorno y Sistemas de Información Contable y financiero en segundo año; Marketing, Gestión de Cadena del Abastecimiento y Taller de Bases de Datos en tercer año; Modelamiento de Negocios, Gestión de RRHH, Estrategia y Taller de Internet en cuarto año; y Taller de Proyecto Informático en quinto año. de los objetivos propuestos en los proyectos, los cuales apuntan a resolver problemas específicos en las empresas.

Debido a lo expuesto, y en el marco del plan de mejoramiento continuo en que se encuentra la Escuela IIE, se planteó la necesidad de formalizar el modelo de trabajo con las empresas que se ha estado llevando a cabo.

El objetivo de este artículo es presentar el modelo o metodología de trabajo resultante y que se ha decidido implementar para formalizar la interacción Escuela/Empresa sobre bases claras y conocidas por todos los actores.

Como consecuencia de la formalización se aspira mejorar la gestión de los proyectos, y por esta vía, lograr un mayor impacto en la calidad de los proyectos desarrollados y en las empresas en que se desarrollan. De esta forma se podrá:

- Uniformar los alcances de los proyectos estableciendo criterios acordados entre los profesores de los distintos módulos;

- Disponer de información homogénea respecto de los proyectos que se llevan a cabo;

- Disponer de evidencias respecto del trabajo realizado en materia de proyectos con empresas;

- Proveer una visión clara, a los actores involucrados (estudiantes, profesionales, profesores y empresarios) respecto de los pasos a seguir para el desarrollo de un proyecto; y

- Certificar los trabajos desarrollados de modo que faciliten la inserción laboral de los estudiantes.

La formalización del modelo de trabajo se presenta mediante las siguientes herramientas:

a) El diagrama de máquina de estados, o de estadotransición, herramienta del lenguaje unificado de modelado (UML) destinada a describir el comportamiento de un objeto, en este caso un proyecto, y que está compuesto principalmente por estados y transiciones, donde una transición lleva al objeto de un estado a otro [17-19];

b) El diagrama SIPOC (Supplier-Input-ProcessOutput-Consumer), herramienta de Six Sigma que permite representar desde los proveedores de las entradas a un proceso, hasta sus salidas y destinatarios o consumidores [20-23]; 
c) El diagrama de procesos en BPMN (Business Process Modeling Notation), destinado a representar la secuencia de procesos que incluye la formalización del modelo de trabajo con empresas y los actores responsables de su ejecución [24].

\section{MODELO DE TRABAJO}

El modelo bajo el cual los estudiantes trabajan en las empresas durante su proceso de formación, supone como objeto de estudio el proyecto que desarrollarán uno o más de ellos bajo la supervisión de un profesor y de un representante de la empresa donde se ejecutará el proyecto. El modelo se diseñó siguiendo los siguientes pasos:

1. Definir los distintos estados posibles del objeto proyecto

2. Definir las actividades que producen los cambios de estado del objeto proyecto

3. Definir la documentación que generan las actividades diseñadas.

Para estos efectos, se establecieron los siguientes estados posibles del objeto proyecto:

- Idea formulada: es aquella que surge de visitas de alumnos y/o profesores a empresas, y/o de reuniones, talleres, seminarios y/o encuentros con empresas;

- Proyecto esbozado: es el que se genera a partir de una idea, y en el que se esbozan sus principales características;

- Proyecto abierto: es el proyecto al cual se ha asignado un profesor responsable de su supervisión y evaluación, con sus requerimientos $\mathrm{y}$ entregables claramente identificados y especificados, al igual que las actividades y los recursos comprometidos por las partes;

- Proyecto asignado: es aquel proyecto con los alumnos asignados y que serán responsables de su ejecución;

- Proyecto formalizado: es el proyecto con la firma de todos los actores implicados (Empresa, Escuela, profesor y alumnos);

- Proyecto ejecutado: es aquel proyecto que ha llegado a término, lo que está respaldado con la disponibilidad de los entregables comprometidos;
- Proyecto evaluado: es el proyecto cuyos entregables han sido evaluados por el profesor responsable de su realización. Como consecuencia de esta evaluación, el proyecto puede ser aprobado para su envío a su validación por parte de la empresa, o bien, ser devuelto a los alumnos para que realicen las correcciones que sean pertinentes;

- Proyecto corregido: es el proyecto que recoge las observaciones efectuadas por el profesor correspondiente para el buen término del proyecto;

- Proyecto validado: es el proyecto que ha sido revisado por la empresa, y que fruto de esta revisión sea aceptado, o bien, requiera correcciones para su aceptación;

- Proyecto cerrado: es el proyecto entregado a plena conformidad de las partes, alumnos, profesores y empresa;

En relación a las actividades que hacen posible los cambios de estado, ellas se definen como sigue:

- Formular ideas: es la actividad -reunión, entrevista, seminario, taller, visita, etc.- en la que participan empresarios, profesionales, profesores y/o alumnos, que pueda dar por resultado una o más ideas suscritas por parte de las empresas, que pueden conducir a proyectos destinados a ser desarrollados;

- Esbozar proyectos: es la actividad bajo responsabilidad de la Escuela conducente a determinar la viabilidad de los proyectos por la vía de describirlos, estimar sus alcances, estimar sus actividades y los recursos que demandará, al igual que los módulos de la carrera que involucrará;

- Abrir proyectos: es la actividad conducente a identificar las actividades y recursos comprometidos por la Escuela y las empresas en las que se desarrollarán los proyectos, especificar los entregables y fechas comprometidas, junto con especificar su alcance, el módulo en el que se desarrolla un proyecto en particular, y el profesor responsable de supervisarlo. La apertura de un proyecto en particular debe ser consecuencia de una fuerte interacción entre la Escuela y la empresa, dando origen a un expediente del proyecto;

- Asignar proyectos: es la actividad destinada a identificar el alumno, o grupo de alumnos 
por parte del profesor del módulo en que se enmarca el proyecto;

- Formalizar proyectos: es la actividad que protocoliza formalmente los compromisos contraídos por las partes (Empresa y Escuela) a través de sus respectivos representantes;

- Ejecutar proyectos: es el conjunto de actividades que han de realizar los alumnos para llevar a buen término los proyectos bajo el monitoreo y supervisión establecidos por los profesores implicados. La evidencia del término de la ejecución de los proyectos es el conjunto de minutas que registran las actividades efectuadas y los entregables comprometidos, documentos que habrán de encontrarse en los respectivos expedientes. El inicio de la ejecución de los proyectos no requiere necesariamente que éstos se encuentren formalizados;

- Evaluar proyectos: es la actividad que realizan los profesores responsables de la supervisión de los proyectos, conducentes a su validación por parte de la empresa; en caso que un proyecto merezca observaciones, éstas deberán ser informadas a los alumnos para su corrección. Esta actividad solo puede realizarse una vez que el proyecto se encuentre formalizado, y la evaluación final debe registrarse en el correspondiente expediente;

- Corregir proyectos: es la actividad que llevan a cabo los alumnos destinada a acoger las observaciones resultantes de las evaluaciones efectuadas por los profesores y orientadas a obtener una evaluación final positiva;

- Validar proyectos: es la actividad que realizan los representantes de las empresas, conducente a la aprobación de los entregables recibidos y que fueron comprometidos formalmente. En caso que existan observaciones, éstas se harán llegar al profesor responsable para que sean acogidas;

- Cerrar proyectos: es la actividad conducente al cierre de todos los proyectos que en su momento fueron abiertos y que se encuentran validados a plena conformidad de las partes involucradas -empresas, escuela, profesores y alumnos-.

Para formalizar el modelo de trabajo a implementar, se definieron los siguientes documentos:

1. Formulario presentación de la idea de proyectos que incluya la descripción del problema/ oportunidad que se aspira encarar, el objetivo que se aspira alcanzar y los productos o entregables esperados, junto con otros antecedentes de interés

2. Convenio específico de respaldo para la ejecución de un proyecto que constituye la formalización del acuerdo de desarrollo de un proyecto en particular establecido entre la Escuela, representada por su director, la empresa mediante un representante de ella, el profesor que supervisará el proyecto y los alumnos que lo ejecutarán; incluye los recursos, actividades y entregables comprometidos entre las partes para la ejecución del proyecto. El propósito del convenio específico es identificar los compromisos adquiridos por las partes.

3. Formulario de evaluación del proyecto que incluye la evaluación que efectúe el profesor responsable de la supervisión del proyecto, la que se habrá de basar en los entregables, en términos de calidad y cumplimiento de las fechas de entrega comprometidas.

4. Formulario de validación y cierre del proyecto que incluye la evaluación general y recepción conforme de los entregables del proyecto por parte del representante de la empresa.

Estos documentos son complementados por otros orientados a favorecer la recurrencia de proyectos en una misma empresa (convenio marco), a facilitar la identificación de los alumnos ante las empresas en las que realizarán, o aspiran realizar, sus proyectos (carta presentación), y a registrar las actividades realizadas y logros alcanzados en el marco de un proyecto específico (bitácora de minutas).

\section{RESULTADOS}

La formalización del modelo de trabajo en base a proyectos en las empresas que está desarrollando la Escuela de IIE se presenta a través del diagrama de máquina de estados, del diagrama SIPOC y del diagrama de procesos en la notación de modelamiento de procesos de negocios (BPMN).

La secuenciación de los estados definidos, junto con las actividades que conducen a dichos estados es desplegada en la Figura 1 mediante un diagrama de 
máquina de estados. Esta figura es complementada por la Tabla 1, donde un diagrama a 5 columnas permite identificar las entradas de cada una de las actividades (procesos), junto con sus proveedores, al igual que las salidas y sus consumidores. Finalmente, en la Figura 2, se presenta el diagrama de procesos en BPMN que permite visualizar tanto los actores implicados, como las condiciones y la secuenciación de cada uno de los procesos (actividades) que conforman el desarrollo de los proyectos.

\section{CONCLUSIONES}

El modelo de trabajo con empresas planteado, constituye una suerte de línea de base destinado a internalizar distintos estilos, formas de trabajo que se llevan a cabo en la actualidad sin un ordenamiento específico y se da una señal a los estudiantes de la existencia de protocolos claros que en una etapa de formación como en el que se encuentran es de gran relevancia.

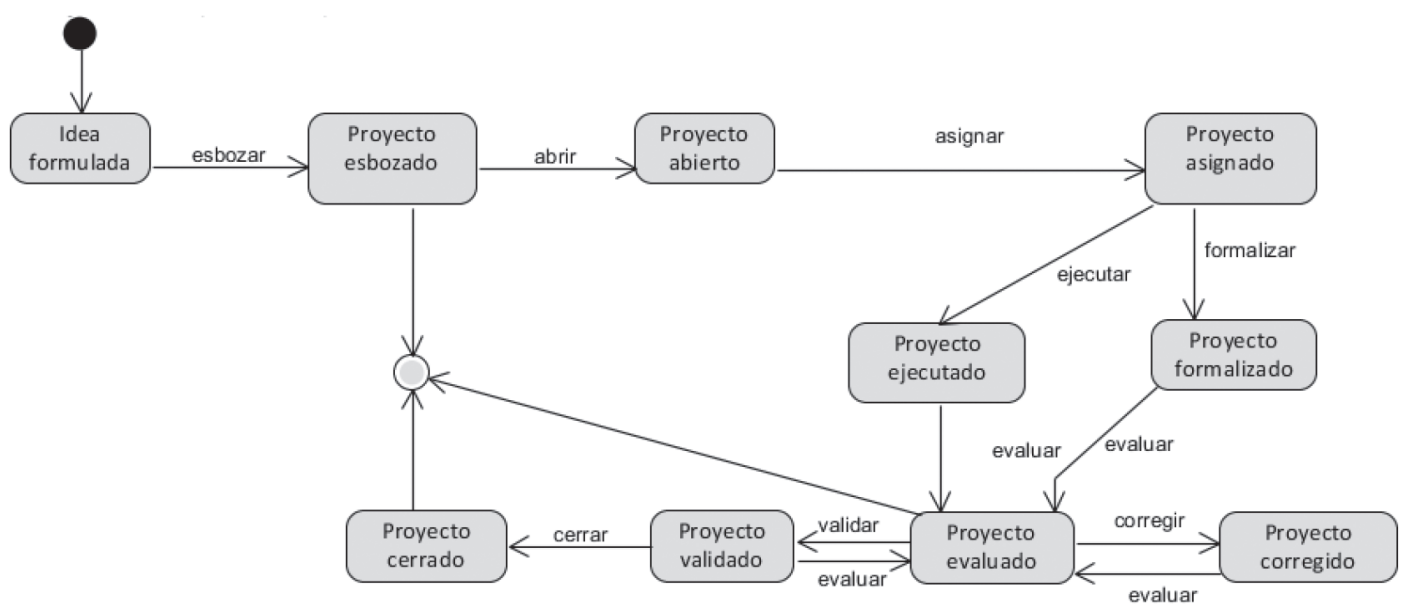

Figura 1. Diagrama de máquina de estados del proyecto.

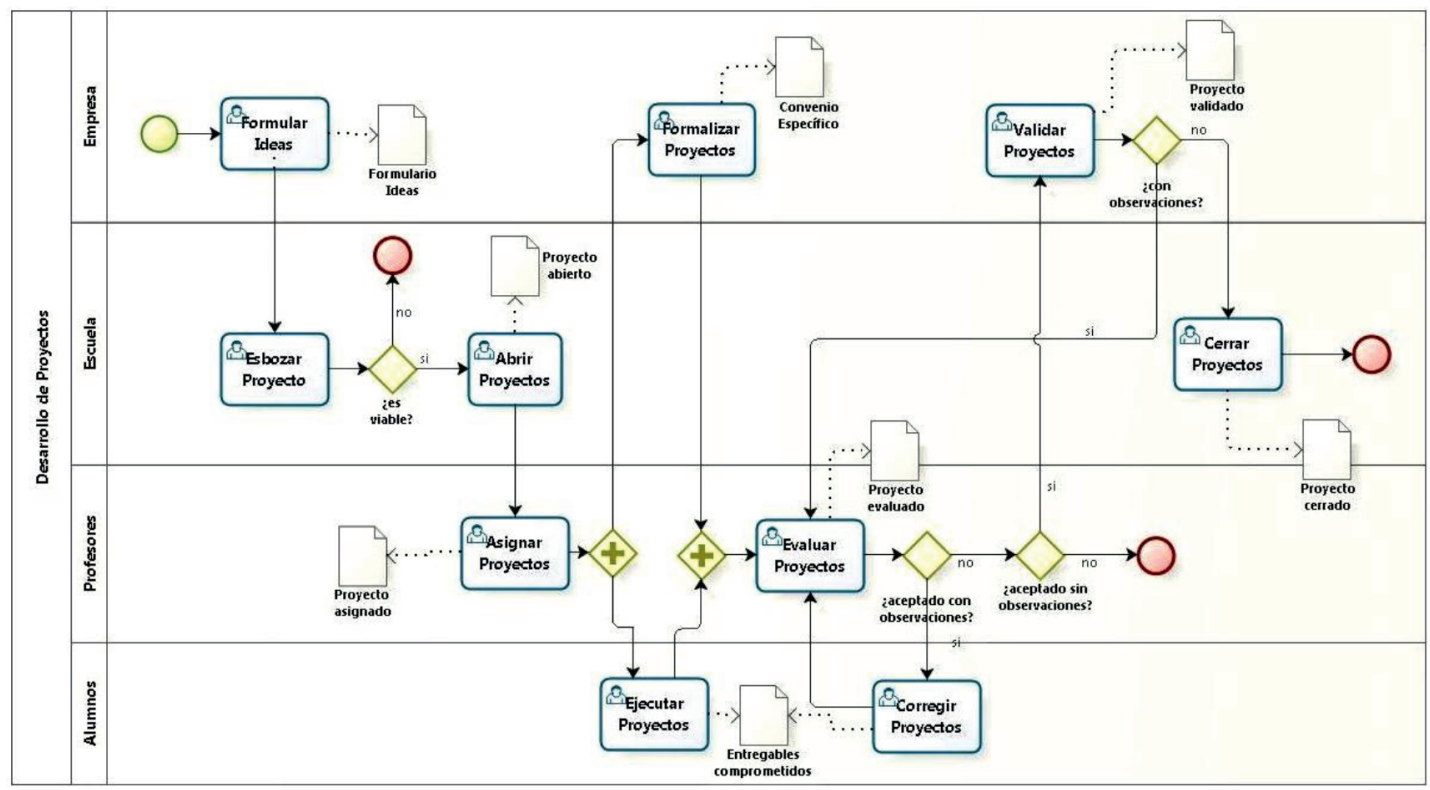

Figura 2. Diagrama de procesos BPMN. 
Tabla 1. Diagrama SIPOC.

\begin{tabular}{|c|c|c|c|c|}
\hline $\mathbf{S}$ & I & $\mathbf{P}$ & O & $\mathrm{C}$ \\
\hline $\begin{array}{c}\text { Alumno } \\
\text { Profesor } \\
\text { Profesional } \\
\text { Empresario }\end{array}$ & $\begin{array}{c}\text { Formulario } \\
\text { presentación de } \\
\text { idea }\end{array}$ & $\begin{array}{l}\text { Formular } \\
\text { idea }\end{array}$ & $\begin{array}{c}\text { Idea } \\
\text { formulada }\end{array}$ & $\begin{array}{l}\text { Escuela } \\
\text { Profesor }\end{array}$ \\
\hline Escuela & $\begin{array}{c}\text { Idea } \\
\text { formulada }\end{array}$ & $\begin{array}{l}\text { Esbozar } \\
\text { proyecto }\end{array}$ & $\begin{array}{l}\text { Proyecto } \\
\text { esbozado }\end{array}$ & Escuela \\
\hline Escuela & $\begin{array}{l}\text { Proyecto } \\
\text { esbozado }\end{array}$ & $\begin{array}{c}\text { Abrir } \\
\text { proyecto }\end{array}$ & $\begin{array}{l}\text { Proyecto } \\
\text { abierto }\end{array}$ & Escuela \\
\hline Escuela & $\begin{array}{l}\text { Proyecto } \\
\text { abierto }\end{array}$ & $\begin{array}{l}\text { Asignar } \\
\text { proyecto }\end{array}$ & $\begin{array}{l}\text { Proyecto } \\
\text { asignado }\end{array}$ & $\begin{array}{l}\text { Profesor } \\
\text { Alumno } \\
\text { Escuela }\end{array}$ \\
\hline $\begin{array}{l}\text { Escuela } \\
\text { Profesor }\end{array}$ & $\begin{array}{l}\text { Proyecto } \\
\text { asignado }\end{array}$ & $\begin{array}{c}\text { Formalizar } \\
\text { proyecto }\end{array}$ & $\begin{array}{c}\text { Proyecto } \\
\text { formalizado }\end{array}$ & $\begin{array}{l}\text { Escuela } \\
\text { Profesor } \\
\text { Empresa } \\
\text { Alumno }\end{array}$ \\
\hline Escuela & $\begin{array}{l}\text { Proyecto } \\
\text { asignado }\end{array}$ & $\begin{array}{l}\text { Ejecutar } \\
\text { proyecto }\end{array}$ & Proyecto ejecutado & Profesor \\
\hline Profesor & $\begin{array}{l}\text { Proyecto } \\
\text { formalizado y } \\
\text { ejecutado }\end{array}$ & $\begin{array}{l}\text { Evaluar } \\
\text { proyecto }\end{array}$ & $\begin{array}{l}\text { Proyecto } \\
\text { evaluado }\end{array}$ & $\begin{array}{l}\text { Alumno } \\
\text { Empresa }\end{array}$ \\
\hline Alumno & $\begin{array}{l}\text { Proyecto } \\
\text { evaluado }\end{array}$ & $\begin{array}{l}\text { Corregir } \\
\text { proyecto }\end{array}$ & $\begin{array}{l}\text { Proyecto } \\
\text { corregido }\end{array}$ & Profesor \\
\hline $\begin{array}{l}\text { Alumno } \\
\text { Empresa }\end{array}$ & $\begin{array}{l}\text { Proyecto } \\
\text { evaluado }\end{array}$ & $\begin{array}{l}\text { Validar } \\
\text { proyecto }\end{array}$ & $\begin{array}{l}\text { Proyecto } \\
\text { validado }\end{array}$ & $\begin{array}{l}\text { Escuela } \\
\text { Profesor } \\
\text { Alumno }\end{array}$ \\
\hline Empresa & $\begin{array}{l}\text { Proyecto } \\
\text { validado }\end{array}$ & $\begin{array}{c}\text { Cerrar } \\
\text { proyecto }\end{array}$ & $\begin{array}{l}\text { Proyecto } \\
\text { cerrado }\end{array}$ & $\begin{array}{l}\text { Escuela } \\
\text { Profesor } \\
\text { Alumno }\end{array}$ \\
\hline
\end{tabular}

Por otra parte, con este modelo se aspira potenciar la red de empresas, así como el proceso de formación de los estudiantes, junto con estrechar la vinculación de la Escuela, y la Universidad, con el medio.

Normalmente, la formalización suele asociarse a un mayor nivel burocrático en las organizaciones, sin embargo, en el curso del desarrollo del modelo, se tuvo cuidado de evitar incurrir en etapas que no agregan valor, asegurándose que cada etapa involucrada sea estrictamente necesaria, y por tanto agregue valor al proceso propiamente tal.

Las primeras promociones de egresados de IIE han señalado que la principal razón por la cual se han logrado insertarse temprana, y exitosamente en el medio laboral, se explica por el modelo de trabajo con empresas bajo el cual fue creada la carrera, aun cuando este modelo no se encontraba formalizado.
Con la formalización del modelo se avanza hacia una fase de consolidación de una forma de trabajar y que está orientada a darle una mayor sustentabilidad, dada por un mayor impacto esperado en términos del aporte de los entregables de los proyectos ejecutados por los alumnos como de la valorización por parte del mercado a estos últimos una vez egresados.

Para los alumnos, egresar con experiencia laboral, constituye un factor que los beneficia en forma importante, al igual que para las empresas, la posibilidad de disponer de recién egresados con algún grado de experiencia laboral formalizada.

Con miras a facilitar la implementación de este modelo de trabajo formalizado, se están dando los primeros pasos conducentes a la construcción del sistema de información que sea capaz de: 
a) registrar la información asociada a los proyectos, empresas, alumnos, módulos y profesores que el modelo de trabajo demanda;

b) efectuar un seguimiento del estado de avance de los proyectos de parte de los profesores de los módulos y la dirección de la Escuela; y

c) satisfacer los requerimientos de información de los distintos actores en apoyo a la operación y gestión de los proyectos.

\section{REFERENCIAS}

[1] R. Schmal. "Un nuevo profesional para la gestión organizacional". Revista del Instituto Internacional de Costos. Edición Especial XII Congreso Internacional de Costos, pp. 87-9. Abril, 2012.

[2] J. Carreras y P. Perrenoud. "El debat sobre las competencies en l'ensenyament universitari". Institut de Ciencies de l'educacio (ICE). Universitat de Barcelona. Barcelona, España. 2005.

[3] F. Esteve, J. Adell y M. Gisbert. "El laberinto de las competencias clave y sus implicaciones en la educación del siglo XXI". II Congreso In ternacional Multidisciplinar de Investigación Educativa (CIMIE). TarragonaEspaña. 4 y 5 de julio 2013.

[4] A. Diaz-Barriga. "Construcción de programas de estudio en la perspectiva del enfoque de desarrollo de competencias". Perfiles Educativos, Vol. 36 N $^{\circ} 143$, pp. 142-162. Enero 2014.

[5] R. Schmal, y A. Ruiz-Tagle. "Una metodología para el diseño de un currículo orientado a las competencias". Ingeniare. Revista chilena de ingeniería. Vol. $16 \mathrm{~N}^{\circ}$ 1, pp. 147-158. Junio 2008.

[6] A. Gohringer. "University of Cooperation Education-Karlsruhe: The Dual System of Higher Education in Germany". Asia-Pacific Journal of Cooperative Education. Vol. 3 $\mathrm{N}^{\mathrm{o}}$ 2, pp. 53-58. 2002.

[7] M. Gessler. "La formación profesional en Alemania". Institut Techni und Bildung, Bremen-Alemania. 2007. URL: http:// www.blogcanaleducacion.es/wp-content/ uploads/2012/11/La-FP-en-Alemaniablogcanaleducacion1.pdf. Fecha de consulta: 30 de junio 2014.
[8] M. Haddara and H. Skanes. "A reflextion on cooperative education: from experience to experiental learning". Asia-Pacific Journal of Cooperative Education. Vol. $8 \mathrm{~N}^{\circ} 1$, pp. 67-76. 2007.

[9] M.L. Rodriguez. "Orientación Profesional y formación basada en el trabajo: conceptos básicos y sugerencias para la intervención". XXI Revista de Educación. Nº 9, pp. 15-33. Huelva, España. 2007.

[10] M. Drysdale and M. McBeath "Self-concept and tacit knowledge: differences between cooperative and non-cooperative education students". Asia-Pacific Journal of Cooperative Education. Vol. 13 No 3, pp. 169-180. 2012.

[11] M. Drysdale and M. McBeath. "Exploring hope, self-efficacy, procrastination, and study skills between cooperative and noncooperative education students". Asia-Pacific Journal of Cooperative Education. Vol. 15 $\mathrm{N}^{\circ}$ 1, pp. 69-79. 2014.

[12] F.J. Montoya y J.J. Aguilar. "La Relación Universidad-Empresa en las Prácticas Empresariales: un Modelo Conceptual desde las Técnicas de Generación de Ideas". Journal of Technology Management \& Innovation. Vol. 8, pp. 196-208. Febrero 2013.

[13] C. Thieme. "El desarrollo de competencias de empleabilidad en dos universidades chilenas. Un estudio empírico". Revista OIKOS, Vol. $11 \mathrm{~N}^{\mathrm{o}}$ 24, pp. 47-72. Diciembre 2007.

[14] M. Marzo, M. Pedraja y P. Rivera. "Un modelo de relaciones empresa-universidad". Revista Europea de Dirección y Economía de la Empresa, Vol. 17 № 1, pp. 39-56. Enero 2008.

[15] C. Aliaga y A. Schalk. "E2: Empleabilidad temprana y emprendimiento. Dos grandes desafíos en la formación superior en Chile". Calidad en la Educación. Vol. 33, pp. 319337. Diciembre 2010.

[16] V. Nocetti. "Rompiendo los paradigmas en la educación y formación profesional de ingenieros: la experiencia de consultorías en Ingeniería Informática Empresarial de la Universidad de Talca". XXVI Congreso Chileno de Educación en Ingeniería. Viña del Mar, Chile. Octubre 2013.

[17] I. Jacobson, G. Booch y J. Rumbaugh. "El proceso unificado de desarrollo de software". Pearson Educación, S.A. Madrid, España. ISBN: 84-7829-036-2. 2000. 
[18] OMG. "OMG Unified Modeling Language Specification". Object Management Group. Disponible en http://www.omg.org/UML/. Fecha de consulta: 17 de Diciembre de 2014.

[19] A. Muneton, C. Zapata y F. Arango. "Reglas para la generación automática de código definidas sobre metamodelos simplificados de los diagramas de clases, secuencias y máquinas de estado de UML 2.0". Dyna. Vol. 74 N$^{\circ}$ 153, pp. 267-283. Sep-Dic, 2007.

[20] K. C. Dias y J. Palma. "Caracterizacao e avaliacao de procesos de selecao de residuos solidos urbanos por meio da tencica de mapeamento". Eng. Sauit Ambient. Vol. 15 No 4, pp. 347-356. Octubre, 2010.

[21] M. M. Siddh, G. Gadekar, G. Soni and R. Jain. "Lean Six Sigma Approach for Quality and Business performance". Global Journal of Management and Business Studies. Vol. 22 $\mathrm{N}^{\circ}$ 2, pp. 589-594. Junio 2013.

[22] H. Felizzola. y C. Luna. "Lean Six Sigma en pequeñas y medianas empresas: un enfoque metodológico". Ingeniare. Revista chilena de ingeniería. Vol. $22 \mathrm{~N}^{\circ}$ 2, pp. 263-277. Abril 2014.

[23] C. Alonso-Torres. "Orientaciones para implementar una gestión basada en procesos". Ingeniería Industrial. Vol. $35 \mathrm{~N}^{\circ} 2$, pp. 159171. Mayo 2014.

[24] J. Freund, B. Rucker y B. Hitpass. "BPMN 2.0 Manual de Referencia y Guía Práctica”. Segunda edición. Departamento de Informática. Universidad Técnica Federico Santa María, pp. 283. Santiago, Chile. 2012. 